\title{
Gradhiva
}

GRADHI

Revue d'anthropologie et d'histoire des arts

5 | 2007

Sismographie des terreurs

\section{Le spectre des génocides}

Traumatisme, muséographie et violences extrêmes

The spectre of genocide. Trauma, museography and extreme violence

Jackie Assayag

\section{OpenEdition}

\section{Journals}

Édition électronique

URL : http://journals.openedition.org/gradhiva/658

DOI : $10.4000 /$ gradhiva.658

ISSN : 1760-849X

Éditeur

Musée du quai Branly Jacques Chirac

Édition imprimée

Date de publication : 1 mai 2007

Pagination : 6-25

ISBN : 978-2-915133-55-4

ISSN : 0764-8928

Référence électronique

Jackie Assayag, « Le spectre des génocides », Gradhiva [En ligne], 5 | 2007, mis en ligne le 12 juillet 2010, consulté le 01 mai 2019. URL : http://journals.openedition.org/gradhiva/658 ; DOI : 10.4000/ gradhiva.658

Ce document a été généré automatiquement le 1 mai 2019.

(c) musée du quai Branly 


\section{Le spectre des génocides}

Traumatisme, muséographie et violences extrêmes

The spectre of genocide. Trauma, museography and extreme violence

Jackie Assayag

«Ce n'est pas la floraison de l'été qui nous attend, mais tout d'abord une nuit polaire, glaciale, sombre et rude. "

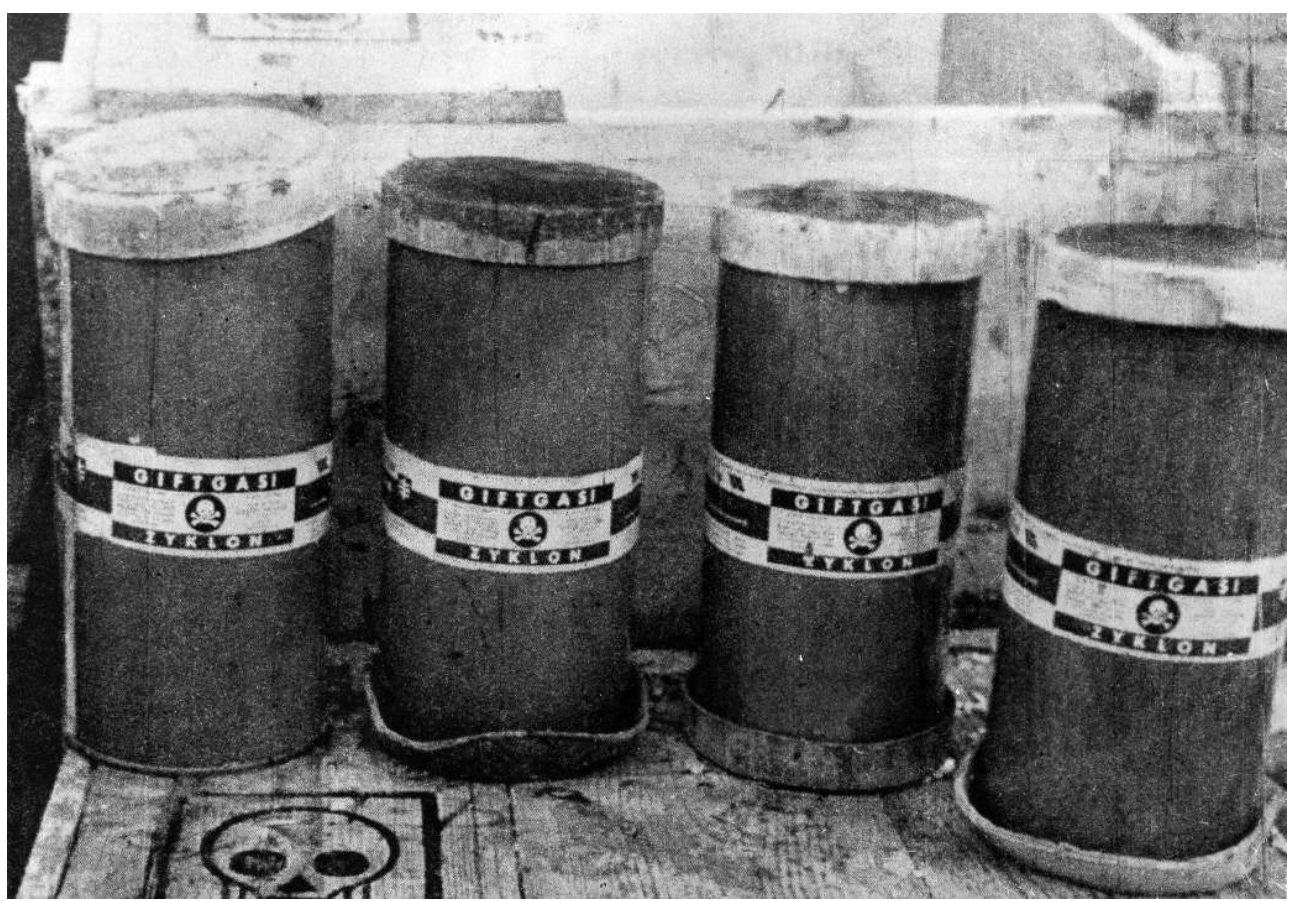

Souhaitant présenter l'un des exemples les plus achevés des créations de l'Antiquité grecque, les conservateurs du Metropolitan Museum de New York choisirent d'exposer une œuvre d'Euphronios, pionnier et virtuose de la technique de poterie dite à « figures rouges » - synecdoque de la civilisation. Ce faisant, c'était oublier «qu'il n'y a pas de 
document de civilisation qui ne soit simultanément un document de la barbarie », comme l'a rappelé Walter Benjamin. Ce qui explique pourquoi l'historien Raoul Hilberg aurait aimé voir présentée une boîte unique, posée sur un socle dans une petite salle de musée, sans rien d'autre entre les quatre murs, afin de symboliser l'Allemagne d'Adolf Hitler: cette boîte de Zyklon avec lequel on tuait les Juifs à Auschwitz et à Maïdanek synecdoque de la destruction des Juifs d'Europe ; «monade de cristal » de l'événement total.

2 Ce que l'historien aurait ainsi donné à voir au public, c'est l'« œil de l'histoire », comme on dit l'œil du cyclone, une zone de perturbation violente et unique dont les effets dévastateurs perdurent longtemps après les faits. Soit une représentation minimaliste mais intensive de l'histoire, vue comme une anamorphose selon un dispositif qui signe la vocation de l'observateur curieux: rendre visible ce qui est caché par les acteurs de l'histoire. Car les génocides ou les violences extrêmes sont des «événements " qui se veulent sans témoins. Le désastre s'avère alors d'autant plus absolu que la scène oblitère ses acteurs et n'autorise aucun regard ; simultanément, les archives et toutes les traces de l'horreur sont effacées. Tout conspire à rendre invisibles à la fois les victimes et leur anéantissement. "On vous assassinera sans reste et sans mémoire ", semblent dire les bourreaux. L'oubli ou le refoulement de l'extermination fait partie de l'extermination, telle une entreprise de disparition généralisée.

\section{Faire voir, rendre lisible}

3 Pendant la Seconde Guerre mondiale, tout se passe comme si l'Europe et le monde occidental refusaient de porter le regard sur l'événement monstrueux que furent les génocides, les massacres administratifs et les " violences extrêmes ${ }^{1}$ ". Mais, dès 1945, une nouvelle époque de la preuve visuelle s'ébauche par le moyen de «la pédagogie par l'horreur ». Pour juger les crimes contre l'humanité commis par les nazis durant la Seconde Guerre mondiale, les alliés ont constitué un tribunal international en forme de procès-spectacle à destination du monde (libre) dans la ville de Nuremberg. À cette innovation majeure, ils ont ajouté, sous l'influence des Américains, deux expériences inédites : présenter des images animées comme preuves à l'audience ; filmer les sessions du procès pour le constituer en archive historique (Delage 2006). Les magazines du monde entier, comme les actualités filmées, relayèrent de façon intense ce déroulé d'images sous l'espèce d'une "épiphanie négative ». Mais la critique des "photos-chocs ", dénoncées dès les années 1950 (notamment par Roland Barthes dans Mythologies), avec leur façon de « surconstruire » l'horreur, aboutissent finalement à l'occulter. Impossibilité de regarder la réalité en face? Impossibilité de se représenter ce qui dépasse l'imagination? Impossibilité d'en assumer la culpabilité, comme certains le suggèrent ? Impossibilité plus grande encore de croire ce qui excède l'humain?

Durant les années les années 1970, la tendance s'inverse lentement, notamment aux É tats-Unis, quand bien même les "négationnistes » et les "assassins de la mémoire » fourbissent leurs armes après la diffusion massive de la série télévisée Holocauste. La mise en spectacle du génocide prend son essor et celui-ci se transforme en langage et en objet de consommation «médiatique ». Depuis lors, d'aucuns considèrent que l'on assiste à une «industrialisation » de la Shoah (Finkelstein 2001) et que nous sommes entrés dans une époque d'institutionnalisation de la mémoire à marche forcée. En l'espace d'une génération, l'Holocauste a quitté les marges pour s'installer au centre de la conscience 
(américaine), pour devenir à la fois omniprésent et paradigmatique des désastres de notre temps (Novick 2001). L'écrivain rescapé Imre Kestész, lui-même, remarque qu'« un conformisme de l'Holocauste s'est formé, de même qu'un sentimentalisme, un canon de l'Holocauste, un système de tabou et son langage rituel des produits de l'Holocauste pour la consommation de l'Holocauste ${ }^{2} »$.

Dans le dessein de contrer la spectacularisation de l'Holocauste, Georges Didi-Huberman propose d'opposer aux « images-écrans » les "images-déchirures », celles "qui laissent filtrer un éclat de réel ${ }^{3} »$. Celui qui les regarde est regardé par elle, de telle manière qu'elles conservent leur "inquiétante étrangeté ", à l'instar de l'image horrible de la Gorgone dont il ne faut jamais regarder la face directement, mais par l'entremise du reflet dans un bouclier poli, sous peine de sidération et de pétrification. Pour sa part, George Steiner emprunte l'expression de Paul Celan «du dedans de la langue-de-mort ellemême » afin de suggérer qu'elle seule restitue par les mots le sens d'" une déchirure de l'histoire à partir de la souffrance qui a marqué les victimes, un exil qui se confond avec le deuil »(Steiner $1987: 16$ ).

Entre-temps, une injonction a pris force de loi : «Plus jamais ça!» Ledit «devoir de mémoire » s'impose. Non seulement cela ne devrait jamais plus se répéter, mais nul ne peut et ne doit oublier ce qui s'est passé. Mais que faut-il faire pour qu'il soit possible d'agir au-delà de cette formule qui s'use au fil du temps? De quoi doit-on se souvenir pour qu'il nous soit possible d'intervenir avant que ne se déploie une nouvelle catastrophe? De quels outils a-t-on besoin pour faire connaitre, faire comprendre, prévenir les crimes liés aux racismes et à l'antisémitisme? De fait, il n'y a pas de réponse claire et définitive à cette question, même si les survivants et leurs descendants, ainsi que les résistants ou les militants anti-totalitaires, invitent aux commémorations et autres célébrations en divers lieux de "mémoires meurtries ». Sur les lieux des crimes ou dans les musées, mais également autour de dispositifs ad hoc, on aménage des espaces propices en vue d'organiser des manifestations collectives ou solitaires aux allures de rituel du souvenir. On édifie aussi des bâtiments destinés à des cultes mémoriels, religieux ou civils, rendus à un nom ou à un événement, plus simplement une photographie ou une image déposée sur un quelconque support, tel un reliquaire, qu'il soit bricolé ou structuré, plutôt discret ou ostentatoire. Ce mémorial impose une esthétique - abstraite ou figurative, difforme ou ciselée - et une atmosphère - sonore ou silencieuse, méditative ou déprimante, ou à intention pédagogique ou «épidictique ». L'essentiel est d'installer une balise ou de tirer un signal d'alarme.

7 L'urgence et l'évidence contraignent à symboliser. On bricole, fabrique, manufacture, configure le «trou noir » et fixe le souvenir, on suspend ou on accroche le traumatisme à un dispositif ou à une scénographie via la narration. Le mémorial réveille la souffrance et approfondit l'absence. L'architecture du vide ou l'esthétique de l'évanouissement le disputent au kitsch. Dans son article sur la «culture iconoclaste des camps » de deux écrivains survivants, Imre Kertész et Ruth Flüger, dont le premier n'y voit qu'une "superstition » attachée aux fantômes à réveiller tandis que le second y reconnaît une scénographie de l'« apocalypse du faux », Catherine Coquio montre que cette divergence permet de distinguer l'art et le kitsch, l'humain et l'inhumain, du moins tels qu'ils se manifestent dans les formes culturelles de la mémoire, tandis que l'art se décompose et que le tourisme bat son plein.

Qu'il y ait kitsch ou non, la question se pose à chaque fois du réglage et de la bonne distance du dispositif, mais aussi celle de sa forme et de sa matière, fût-il informe ou 
bizarre, oblique ou frontal, monstrueux ou répulsif, massif ou évanescent ; un « je ne sais trop quoi » ou un " presque rien » peut faire l'affaire. Après tout, un quelconque artefact est susceptible de cristalliser une émotion, d'exposer une signification, de suggérer une norme éthique sur la base d'un "régime de représentation» qui le calibre et fait raisonner celui qui le regarde. Selon Stephen Greenblatt, la « résonance », c'est le pouvoir qu'a l'objet exposé d'aller au-delà de ses frontières formelles pour atteindre un monde plus large, d'évoquer pour celui qui le regarde la complexité et la dynamique des forces culturelles desquelles il a émergé et pour lesquelles il mérite vraiment d'être vu (1991). Mais l'objet exposé a aussi le pouvoir («merveilleux») d'arrêter la vision par son empreinte forte, son caractère unique et saisissant qui impose une attention soutenue ${ }^{4}$. Du croisement inédit entre "résonance » et « merveille » naît parfois le «terrible » sous forme de trace ou d'image, d'icône ou de montage, de miroir ou d'un quelconque bouclier sur lequel se réfracte la vision de l'« intolérable », sous l'espèce d'une figuration de la reconnaissance du semblable et du différent.

Certes, la vision creuse le désarroi et augmente la douleur, comme s'accrôit l'incompréhension. Mais l'affliction ou la protestation se condensent entre-temps. La face terrible des choses impossibles à voir dans la réalité, nous pouvons en faire l'expérience (amère et superficielle) au regard des tortures et des souffrances incompréhensibles créées par la brutalité étatique - morceaux de chair martyrisée ou de corps fracassés ; montagne de crânes empilés, rangés, disloqués ou abandonnés; cadavres entassés dans les charniers ou squelettes amoncelés ou enchevêtrés - immonde théâtre de la cruauté. Comment peut-on surmonter l'effroi ou l'horreur? En regardant le reflet dans le bouclier. Des voix éteintes, des visages disparus, des images effacées se rappellent à nous en un écho insistant, atone, douloureux, récurrent et peut-être rédempteur... mais pour un temps limité : «L'image vraie du passé passe en un éclair. On ne peut retenir le passé que dans une image qui surgit et s'évanouit pour toujours à l'instant même où elle s'offre à la connaissance ", écrivait Walter Benjamin (2000 : 430).

10 Ainsi se donne à voir, sentir, entendre, comprendre ce que beaucoup prétendent hors de la représentation, tant l'arsenal des sévices dépasse et l'entendement et l'imagination. Rendre sensibles la violence et l'abjection de l'événement, y compris sur un mode métaphorique et sans même évoquer les conséquences traumatiques pour les survivants et leurs descendants, est une façon de configurer l'inimaginable en arrachant une image ou une parole au désastre. Mais rendre lisible l'illisible ou visible l'invisible exige, répétons-le, un montage qui articule une présentation matérielle dont on espère qu'elle comblera la «brèche » entre le passé et le futur. De sorte que s'ouvre le présent du temps contre le poids de l'oubli, contre le poids de ce qui s'est passé parce que "notre héritage n'est précédé d'aucun testament ", selon l'aphorisme poétique de René Char. Il ne s'agit évidemment pas d'expliquer mais de suggérer une forme qui signe la catastrophe et la " survivance ». Ressentir cette expérience dans la temporalité de l'épreuve est justement ce qu'exprimait Kertész dans son discours de réception du prix Nobel, lorsqu'il souligna combien il faut faire d'effort pour ouvrir les yeux face à l'arrivée fatale de l'impossible, qui demeure en nous comme une « plaie ouverte».

11 Cultiver une mémoire commune des horreurs dans le cadre muséographique est une manière de combler la «brèche », de restaurer la dignité en conjurant l'abjection par le biais d'une exposition qui remplit trois fonctions : offrir un espace de parole publique pour que les violences soient dites ou montrées et qu'elles soient imputées publiquement aux uns et aux autres; procurer un enseignement moral au sein de «forums hybrides » 
en faisant entendre, de gré ou de force, le sens des événements passés aux générations qui ne les ont pas vécus ; enfin rendre justice aux victimes en désignant les bourreaux sur une scène qui tient lieu de recréation vivante du désastre national à la suite d'une psychothérapie de groupe élargie ${ }^{5}$. L'entreprise se veut cathartique et thérapeutique dans l'attente que se dévoilent les multiples "perlaborations », c'est-à-dire le travail psychique qui permet d'accepter certains éléments refoulés et de se dégager de l'emprise des mécanismes répétitifs. La visée est également éthique et prophylactique, politique et sociale, parce qu'elle délivre un message de vigilance et d'espoir pour la reconnaissance généralisée du caractère inacceptable d'actes qu'on ne peut supporter ni accepter.

En ce sens, la scénographie muséale s'apparente à un "avertisseur d'incendie ", selon la belle formule de Walter Benjamin. Comme si la vision poétique et politique d'une représentation mémorielle transformait tout un chacun en témoin ex post de la sismographie des terreurs de masse, présentée dans les musées édifiés aujourd'hui un peu partout dans le monde.

\section{Passeurs de désastres et de comparaisons}

13 Le $\mathrm{XX}^{\mathrm{e}}$ siècle fait encore partie des conditions actives de ce moment de transition dans lequel nous vivons et nous pensons. En ce sens, nous sommes contraints de porter notre regard sur les guerres et la paix. Mais nous devons plus encore focaliser sur le complexe catastrophique de la modernité qui a scellé la liaison énigmatique entre nos « sécurités démocratiques » et les « violences extrêmes ». Désormais, tout se passe comme si la vie pacifiée requérait la vision des désastres humains, bien qu'on ne puisse évidemment pas réduire notre condition actuelle aux atrocités qui ont scandé l'histoire contemporaine (Brossat 1996: Avant-propos). Il n'empêche. Les épreuves enténébrées traversées par les générations qui ont vécu ou connu le génocide arménien, le GULag, le nazisme, les totalitarismes, mais aussi Hiroshima et Nagasaki ou, plus récemment, le Timor oriental, le Cambodge, le Rwanda, l'ancienne Yougoslavie et aujourd'hui le Darfour, ne peuvent qu'interpeller ceux qui sont "nés après »; et cela d'autant plus que la liste n'est pas exhaustive. De surcroît, l'emploi sans limites du matériau humain à des fins de guerre ou d'extermination, du travail forcé ou de la négligence criminelle de la déportation sous couvert d'ingénieries sociales messianiques n'a pas disparu de notre horizon d'attente, loin s'en faut.

14 À cause de cette histoire "massacrante » de la modernité, il revient aux practiciens des sciences sociales d'assumer la charge de passeurs des désastres du passé au tournant du $\mathrm{xxI}^{\mathrm{e}}$ siècle où les rangs des rescapés, des acteurs, des bourreaux, des témoins s'éclaircissent. Garantir l'« ininterruption » des récits des violences extrêmes dans les guerres, les conflits et les camps est la responsabilité qui nous échoit, de fait et de droit. Nous sommes requis, sommés, mobilisés d'établir une connaissance raisonnée du mode catastrophique de l'histoire contemporaine, ou plutôt d'une pluralité d'histoires puisque les génocides et les crimes contre l'humanité ne se déploient pas uniquement en Europe. Chacune de ces histoires doit intégrer cette expérience des violences extrêmes pour rendre intelligibles les conflits passés, présents ou à venir ; non seulement parce que cette déchirure affecte notre actualité, mais aussi pour ne pas abandonner le passé naufragé à la mémoire, au rituel et à la commémoration. 
La tâche s'avère d'autant plus nécessaire et difficile que les reliquaires, à la fois maudits et sacrés devant lesquels on vient se recueillir, prier, s'affliger, entretiennent le " réenchantement » religieux de l'histoire « catastrophée ». Ce constat désabusé soulève la question de savoir si les expositions doivent s'évertuer à produire la solidarité mécanique idéale et le consensus, ou l'examen critique des conduites problématiques qui invitent à la discorde. Si l'on peut légitimement se réjouir de la multiplication des musées ou des camps du «tout-mémoire » concernant les «laboratoires de décivilisation » un peu partout dans le monde, on déplore que ces hauts lieux n'inscrivent généralement que la trace et le souvenir. Or la mémoire ou la compassion ne suffisent pas; comme ne suffisent pas les commémorations tant elles restent captives des luttes d'intérêts et des dilemmes du présent. L'injonction de l'interdit de la représentation par les représentants mandatés ou autoproclamés desdites "communautés» ne facilite pas davantage la transparence de l'histoire, au contraire. Ce qu'exemplifie justement la montée en puissance du «judéocide » depuis les années 1970 dans le champ de la perception de la catastrophe, au titre du caractère " unique » et « incomparable », "sans précédent », diton souvent, de la Shoah. Celle-ci se pare alors d'une aura religieuse pour mieux la théologiser et la sacraliser, tel un «mystère » qui fraie la voie au culte qui lui sera voué ; le sentiment d'intolérable n'oblige pourtant pas la conversion à l'ineffable ${ }^{6}$. Quant à la notion d'« incommensurabilité ", elle s'avère confuse, "gênante, et presque incompréhensible» en termes de droit, selon le juriste et sociologue Mark Osiel (2006: 174-175), outre qu'elle accrédite le silence des bourreaux et laisse le champ libre aux « assassins de la mémoire ». Quant à l'érection de monuments et de stèles funéraires sur les lieux même de la "Catastrophe", ils tendent à nous installer au centre d'un récit raisonné de l'histoire des $\mathrm{xx}^{\mathrm{e}}$ et $\mathrm{xxI}^{\mathrm{e}}$ siècles dont le cœur ne bat qu'en Europe. Encore que même en Europe les musées des guerres et des terreurs revêtent des caractéristiques nationales, régionales ou locales à cause des réappropriations conflictuelles de l'histoire et des discontinuités d'expérience dans l'accommodation du passé, comme le montre Sophie Wahnich, qui s'interroge sur le fait que les musées savent transmettre l'effroi mais ne questionnent plus la notion de terreur comme catégorie politique. Dans cette Europe plurielle « déchirée », l'auteur déplore que la pitié et la lamentation supplantent le travail sur les traces résiduelles de la honte.

Quoi qu'il en soit, le judéocide recouvre et "secondarise» désormais l'ensemble des massacres de plus ou moins grande échelle. Devenu la nouvelle religion laïque et catastrophique de notre temps, il se présente comme un énoncé non ouvert à la discussion. Or ce rideau de fumée métaphysique produit un partage signifiant et hiérarchisant envers les autres génocides et les "génocidés des Autres", dont les victimes sont pourtant eux aussi coupables d'«être nés». Forte de la croissance exponentielle et tous azimuts des études sur les génocides dans l'espace et le temps ${ }^{7}$, une brèche s'ouvre dans le discours de l'exception (de l'élection?) à tous ceux qui s'affrontent aux autres « dévisagés » du monde, entendons le monde non européen ${ }^{8}$. Par exemple, la dissociation intimidante d'Auschwitz d'avec les exterminations staliniennes définies comme ne relevant pas de la catégorie de génocide a pour effet de hiérarchiser ce qui ne devrait pas l'être. En éludant tout rapprochement entre le judéocide et les exterminations dites "politicides », on rejette les horreurs du GULag hors de notre histoire ; une histoire d'administration pénitentiaire et de déportation qui a duré plus d'un demi-siècle, intégrant l'usage de la terreur à chaque étape de son fonctionnement; une histoire qui n'a laissé que peu de traces et pas de véritable musée, ni même un inventaire des crimes 
bien que les victimes fussent innombrables, les bourreaux étant qui plus est restés impunis. Élisabeth Gessat-Anstett le souligne lorsqu'elle rappelle que l'activité artistique fut conçue sur le mode de la résistance contre l'outrage de la violence et de la terreur subie.

Un autre exemple de cette dissociation disqualifiante consiste à rejeter la conformité des massacres coloniaux au paradigme du génocide strictement défini par Raphaël Lemkin ${ }^{9}$. Or ce récit autocentré de l'histoire occidentale comme histoire universelle est une nouvelle théodicée de la démocratie perpétuelle (blanche), qui communie dans le souvenir et la commémoration de ladite Shoah, laquelle est devenue l'étalon des génocides.

En dépit des difficultés à nommer la pleine ampleur des génocides et des pratiques de l'extermination, qui s'affichent sous nos yeux sur le ruban déroulé de l'actualité, les cas de la " purification ethnique » serbe et des massacres à grande échelle dans un temps très court (effectués moins à la machette que par les balles et les grenades) au Rwanda ont opéré un changement dans notre champ de perception de la catastrophe. L'article de Célestin Kanimba Misago souligne l'importance que revêtent la pédagogie de l'horreur et «la muséographie traumatisante » pour assurer un avenir meilleur au Rwanda tout en s'attachant à en mettre en évidence les limites, au regard des germes de division sociale et des sentiments ambivalents qui peuvent provoquer des réactions diamétralement opposées dans les différents camps.

Dorénavant, on sait que les partages académiques entre les circonscriptions respectives des génocides ou ethnocides établissent une hiérarchie des crimes dont le paradigme est le judéocide et dont la norme est le droit international, lequel fut conçu dans le cadre de la guerre froide et des puissances rivales entre l'Ouest et l'Est - en escamotant le reste du monde. Dans le champ profus des massacres d'État, il faut donc "provincialiser l'Europe », selon la formule de l'historien « subalterniste » Dipesh Chakrabarty (2000), du moins si l'on veut désoccidentaliser l'approche des violences extrêmes en montrant l'amplitude du phénomène ailleurs qu'en Europe ${ }^{10}$. «Là où est passé l'Européen, la mort semble poursuivre l'aborigène ", comme l'écrivit Charles Darwin, résumant la saga du processus conduit par l'Europe pour civiliser les sauvages. Cette démarche permet de délaisser sans regret la casuistique des intensités diaboliques, tératologiques, morales : la volonté de détruire une pseudo-race n'est ni plus noble ni plus infâme que la volonté de détruire une pseudo-classe ou toute autre catégorie de population dont les membres sont considérés en surnombre. «À quelle balance voudrait-on peser l'extermination par le gaz et l'extermination par le travail [...] et qui tirerait argument du nombre des populations anéanties dans un système ou bien du sadisme des bourreaux dans une autre?» questionnait Claude Lefort (1976:147-148).

20 Notre responsabilité de passeur exige donc de "comparer l'incomparable " (Detienne 2000), autrement dit de confronter les multiples scènes de l'« extrême » sans a priori culturels, moraux ou politiques. La sensibilité aux spécificités génocidaires ne doit pas interdire l'exploration des différences avec tous les autres. Mais ne sont toutefois convoqués dans ce dossier d'enquêtes que l'Europe, l'Afrique du Sud, le Rwanda, le Cambodge, la Russie soviétique, outre quelques excursions ici ou là-bas. 


\section{S'il y a de l'irreprésentable}

21 De l'effacement radical des témoins qu'illustrait le dispositif muséographique imaginé par Raoul Hilberg dans l'introduction, il ne faut nullement déduire que les génocides emportent les marques de l'inhumain, du sublime ou d'on ne sait quel ineffable, sous prétexte que les expériences limites de "ceux qui ont vu la Gorgone ", comme l'écrivait Primo Levi, nous font plonger dans l'horreur ou l'effroi. «Dans ce face-à-face aveugle du visage à tête de mort, qui est le face-à-face avec le sans-regard» (Nancy 2001: 31), l'individu se découvre déconcerté, interloqué, sidéré, médusé, au point que l'effet de confusion le laisse définitivement inconsolé. Le motif si souvent ressassé de l'irreprésentabilité du génocide, c'est-à-dire du caractère à la fois "impensable » et « intraitable », voire transcendant (que la Shoah rend emblématique), doit être récusé. En posant l'«irreprésentable » et par voie de conséquence l'«impensable» au cœur des massacres, on prolonge l'hyperbole spéculative qui entoure d'une même aura mystérieuse de terreur sacrée toutes sortes de violences extrêmes (Rancière 2001). Or cette théologie négative occulte la banalité humaine des massacres de masse et soustrait les motifs concrets qui ont poussé à agir ou à ne pas agir; elle gomme le modus operandi des processus et ignore les explications du phénomène; enfin, elle déporte le fait génocidaire dans la sphère de la seule représentation ou dans le champ de l'épistémologie hypercritique afin d'étayer le "négationnisme ${ }^{11}$ ». Il faut donc rappeler sans cesse que l'atrocité des guerres civiles ou nationales, au service dudit «mal radical», se confond avec l'ordinaire et s'exécute la plupart du temps au rythme d'horaires quotidiens planifiés et d'une division du travail que fixe un encadrement à la fois zélé et compétent. L'activité génocidaire s'épanouit dans l'«extraordinaire normal». La pression de l'environnement hégémonique suffit pour qu'un être-là en situation s'anime et s'arme à la faveur de l'identification au "groupe-en-fusion » dans le cours tumultueux de l'histoire humaine, trop humaine.

Pour contrer ces tentatives "négationnistes ", il faut souligner que les victimes ellesmêmes éprouvent le besoin d'attester d'une « présence » à travers les productions écrites ou artistiques (fort rares évidemment mais présentes jusque sous la cendre [Des voix sous la cendre 2005]). Ceux-là veulent ainsi maintenir coûte que coûte l'image d'un monde extérieur, un monde hors temps et en dehors du camp qui leur permet d'arracher de l'enfer une activité cognitive autonome par le biais de lambeaux de l'esprit ou de la culture; comme si savoir et faire savoir étaient des manières de rester humain. C'est la raison pour laquelle Hannah Arendt place dans la " conscience déchirée », ou plutôt dans la « déchirure historique ", les figures de l'artiste, du poète et de l'historien à l'instar de «bâtisseurs de monuments» sans lesquels l'histoire que les mortels jouent et qu'ils racontent ne survivrait pas un instant (Arendt 1993 : 230). L'intérêt de l'article de Tzvetan Todorov est d'ajouter la figure de l'anthropologue à cette galerie de héros puisqu'il montre que les engagements de Germaine Tillion - une femme non embrigadée, de la Résistance à la guerre d'Algérie en passant par Ravensbrück, et donc témoin de son siècle - ont survécu face à l'extrême grâce à son goût pour l'observation ethnographique participante, son aptitude aux échanges et à la mise à distance entre soi et son expérience, et enfin son savoir, qui lui servit toujours de bouclier.

De cet effacement découle aussi le désir chez d'autres de fixer l'« absence » sous l'espèce d'une «icône de l'horreur », quel qu'en soit le support matériel ou la forme, afin qu'il 
cristallise la mémoire endeuillée et serve de signal d'alarme : une «monstration » en tout cas se fait jour. N'importe quelle marque ou objet devient une production testimoniale qui fixe le mal et son remède, qui comble la béance entre l'hypertrophie du vouloir tout voir et l'enténèbrement qui entoure l'événement de terreur. Certes, le témoignage de l'absence est au-delà de la représentation et en deçà de la mémorisation, comme il échappe d'ailleurs à l'imagination, tant l'image ou le mot défaillent. Certes encore, l'horreur de l'extrême violence défie et l'imagination et l'intelligence. Mais la trace ou l'objet, aussi fragiles soient-ils, peuvent déclencher l'image ou le récit, aussi lacunaires et ambigus soient-ils. C'est dire que la symbolisation du « trou noir » de l'histoire s'impose à ceux qui en ont la volonté et les moyens, et combien ils s'ingénient à l'exercice de la représentation "malgré tout ${ }^{12}$ ", en dépit d'obstacles presque insurmontables. Transmettre le « destin du trauma », qui constitue "a break in the mind's experience of time " (Caruth 1996), ne revient pas à lui ôter figure humaine ni à l'hypostasier dans l'indicible ou l'intimidant. La transmission recouvre, dans la "parole trouée », le fil de l'anéantissement.

À côté donc de ceux qui se murent dans le silence, certains expriment l'exigence de dire ou d'écrire, de nommer ou de fabriquer, voire d'analyser la catastrophe pour la rendre tangible. Ce faisant, ils arrachent la représentation du désastre au travers de lambeaux sortis du néant de victimes à bout de malheur. Ainsi confèrent-ils forme à l'inimaginable au travers de bribes et de restes d'images ou de mots qui ne l'illustrent pas. N'est-ce pas d'ailleurs là où achoppe la pensée que nous devons persister dans la pensée en lui donnant un tour nouveau? Celle d'une forme ou de l'art par le biais d'une présentation qui donne silhouette à l'inimaginable ou rend lisible l'impossible; sachant toutefois, écrivait Maurice Blanchot, qu' 'il y a une limite où l'exercice d'un art, quel qu'il soit, devient une insulte au malheur ». Refuser la figuration ou l'expression revient, en outre, à engloutir l'histoire et à se priver de faire œuvre utile à partir de la mémoire en imposant une morale à l'histoire. La mémoire a une fonction rédemptrice car l'oubli perpétue l'offense du crime.

\section{Histoire et traumatisme}

La révélation de l'Holocauste et des autres génocides qui se sont déroulés au cours $\mathrm{du} \mathrm{xx}^{\mathrm{e}}$ siècle est à la fois une réalité et une question ultime qu'on ne peut esquiver. La face de l'humanité en est sortie "dévisagée ». Cette déchirure a mis en crise la perception banale des Lumières, dont les Modernes portaient le flambeau, pour la rendre en partie ou en totalité désuète. Depuis lors, nous éprouvons une difficulté à penser la finitude de l'être singulier, ou la mort, laquelle fut " administrée » à des millions d'« exemplaires » dans les guerres ou les camps. La mort n'est plus à même d'être donnée à l'individu, ainsi "dépossédé de la dernière chose qui lui restait, et la plus misérable ", comme l'écrit Theodor Adorno. La difficulté de se rapporter à la transcendance qu'on ne parvient ni à affirmer, ni à nier après les génocides, comme la difficulté de réaliser qu'après l'éruption de cette catastrophe qui recouvre non seulement l'extermination perpétrée dans les camps mais aussi « le monde de torture qui a continué d'exister après ", et l'invention de la bombe atomique ou les comptes rendus les plus horribles des multiples guerres, confirment le diagnostic. Les violences extrêmes ont annihilé l'interrogation sur le sens et rendu obsolète et indécente toute philosophie affirmative (Anders 2001). 
26 Mais ce constat - inspiré de « la métaphysique après Auschwitz » selon Adorno (2006) n'a pas détruit la volonté de faire ce qu'interdit la devise des mineurs, à savoir « travailler en s'immergeant profondément dans l'obscurité, sans lampe » - et ne pas y mourir afin d'apercevoir des éclats de réalité dans le cortège d'horreurs et de massacres. En ce sens, les holocaustes $\mathrm{du} \mathrm{xx}^{\mathrm{e}}$ siècle peuvent donc être vus comme une sorte de division et de passage d'époque entre la modernité et la postmodernité. À l'âge du "traumatisme de guerre " succède l'âge du traumatisme de la "Solution finale ». Mais cette dernière est elle-même devenue post-traumatique dans le contexte de l'après guerre froide. La dimension post-traumatique des manières de faire ou de penser fut progressivement liée à un environnement conçu comme devant être protégé et envers lequel on doit prendre moult précautions. Tout se passe comme si nous assistions, en y participant, à une « crise de l'avenir » dont l'idée, souvent vague, est présente chez tous. Le futur construit sur la catégorie du progrès, avec son corollaire qu'est l'optimisme historique, se délite. Aujourd'hui, lorsque nous regardons vers l'avant, nous voyons moins la promesse de lendemains qui chantent qu'un avenir chargé de menaces qui s'accumulent. Notre futur n'est plus le laboratoire où s'ébauchent les grandes œuvres de l'humanité, mais l'atelier où se fabriquent des dispositifs qui peinent à nous protéger, en dépit de nos préventions, des cataclysmes annoncés, comme celui de l'« inhumanisation ».

La question contemporaine du traumatisme s'inscrit dans ce paysage où l'Ange de l'histoire aurait pris congé pour mieux lester notre mémoire. Elle s'est surtout exprimée par le besoin compulsif de "rouvrir » obsessionnellement les plaies de l'histoire. La multiplication des appels aux « mémoires blessées », que les acteurs patrimonialisent afin de s'octroyer des bénéfices secondaires, traduit un changement dans les représentations du devenir des sociétés. À la geste héroïque, qui forgeait antérieurement les communautés victorieuses et conquérantes, succède la plainte de ceux qui se représentent comme les « vaincus de l'histoire ». Cette radicalisation politique fondée sur la «mémoire meurtrie» transforme le passé en ressource stratégique, en l'espèce " capital symbolique ». Ce faisant, l'affirmation du caractère unique d'un traumatisme issu d'un crime passé non reconnu dans l'espace public autorise des allocations de reconnaissance à tous ceux qui promeuvent ou défendent les politiques de la mémoire et de l'identité. L'afflux massif d'acteurs ou de groupes en posture victimaire met en péril la version triomphante qu'orchestre habituellement l'épopée nationale. L'affirmation du caractère unique d'un traumatisme issu d'une ancienne injustice subie invite à réclamer des réparations au titre d'une identité abîmée, d'une mémoire saignante, d' « un passé qui ne passe pas » au sein d'une communauté donnée. Cette conception patrimoniale du passé et de l'identité fait le terreau de la compétition entre les victimes. La tension s'avère d'autant plus forte que les États-nations répugnent généralement à se bâtir sur l'autocritique, le repentir, la culpabilité, le pardon.

Dans la mesure où nous vivons tous dans un contexte postholocauste du « faire vivre et du laisser mourir» (Foucault 1997: 229-230), l'idée prévaut que la culture ou même l'histoire seraient devenues essentiellement traumatiques. Aujourd'hui, on ne travaille plus contre le trauma, mais avec lui par les voies de l'oubli, du déni, du refoulement et du "souvenir écran", sachant que le traumatisme s'invite de toute façon de manière impulsive dans nos cultures en proie à des cataclysmes disparates. En conséquence, une forme de narration à la fois fondatrice et rédemptrice se répand parce qu'elle paraît susceptible de déterrer le souvenir refoulé du trauma historique en forçant les individus à travailler son effet persistant. De cette manière on prévient la compulsion névrotique à 
répéter le passé de façon aveugle et non critique, du moins est-ce de la sorte que Jürgen Habermas le conçoit : «La domination du passé, qui revient comme un cauchemar pour peser sur le présent non réparé, ne peut être repoussée que par la puissance analytique d'une forme de remémoration qui regarde calmement ce qui s'est passé sans le voir en termes moralement neutres. » (1985) Mais c'est aller sans doute trop vite en besogne : l'invitation à oublier une expérience traumatique rappelle nécessairement l'expérience qu'il fallait oublier.

À ce titre nous serions tous devenus des "survivants ». Cette vision postmoderne de l'histoire signe le renversement d'un "régime de véridiction", puisque le soupçon attaché au trauma (dans son ancienne acception experte) se voit supplanté par la reconnaissance de l'universalité de l'être souffrant hic et nunc. Le sentiment se diffuse que l'événement traumatique concerne un nombre croissant d'individus et de populations, sinon dorénavant tout un chacun, sur un large spectre qui va du mal-être existentiel jusqu'au traumatisme de guerre, voire aujourd'hui le terrorisme. Ce retournement est d'autant plus vif que nous éprouvons tous le sentiment de vivre dans un présent qui se caractérise par le fragment et l'excès au sein d'un monde violent, désorienté et en morceaux. Si l'époque tend à valoriser le traumatisme, alors qu'on célébrait hier la figure du héros, c'est parce que l'emportent maintenant la fidélité et le respect envers toutes les victimes. De là ce désir croissant de partager avec elles ou de vouloir s'identifier à elles sur un mode compassionnel.

De fait, la généralisation sans distinction de la catégorie de "survivant», ainsi que la coalescence de la culture ou de l'histoire avec le traumatisme, comme la fixation sur le "trauma» et la fréquence du passage à l'acte, ne manquent pas de produire des distorsions dans l'histoire et d'obscurcir les frontières géographiques et historiques, éthiques ou politiques. Par exemple, à côté de la prise de conscience controversée des génocides et du caractère traumatique des violences extrêmes, d'autres conflits, terreurs et massacres de masse ont été requalifiés "génocides", y compris pour les époques antiques, prémodernes et modernes. Ces incriminations rétrospectives sont majoritairement le fait de descendants (autoproclamés) de population décimées. Dans la mesure où les individus et les collectivités se retournent volontiers vers leur passé (fréquemment réinventé) pour définir leur identité, il n'est pas rare qu'ils découvrent des phases traumatiques dans leur histoire, qui légitiment à leurs yeux le sentiment d'être une victime. Cette quête généalogique (souvent imaginaire) favorise les incriminations ex post de génocides et conforte l'idée que le groupe auquel on appartient est singulier et incommensurable. Au sein de groupes variés - ethnies, tribus, "races », communautés, peuples (dit autochtones), religions ou nations - tend à se développer ladite "concurrence des victimes» en vue de l'obtention du palmarès de la souffrance, d'allocations de reconnaissance ou de dividendes afférents (Chaumont 1997). Inspirés par le «narcissisme de la petite différence », les « enracinés » de tout poil en appellent alors à la retrempe de la « race » et au «ré-enchantement » de la communauté/nation en péril qui doit retrouver son intégrité : « C'est à moi et c'est mon droit car j'étais là avant »! Or «toute idéologie qui investit les victimes d'une autorité particulière pour formuler des exigences à l'égard des autres, qui n'étaient pas eux-mêmes les agents du dommage qui leur a été causé, frappe par son incohérence», comme l'argumente Mark Osiel (2006: 227).

31 La notion de traumatisme sert aujourd'hui à définir une identité collective qui se confond dans une essence à la fois personnelle et collective. L'idée d'une mémoire toujours en 
souffrance est devenue une manière de fabriquer une identité qui s'avère cependant impossible à formuler clairement, sinon sous l'acception abstraite et discutable de « victime ». Cette invocation sert à légitimer une identité politique qu'il serait difficile de justifier par tout autre critère que celui-là. Les membres des cultures qui ont traversé de près ou de loin une expérience malheureuse commune, ou qui ont contribué à l'édification et à la perpétuation d'un héritage prestigieux, s'avèrent extrêmement sensibles à propos de faits ou d'états spécifiques que les autres ne partagent pas.

L'insistance sur le caractère unique d'un traumatisme subi par telle ou telle population, alors qu'il n'existe pas d'étalon de mesure de la souffrance, revient in fine à négliger ou nier les massacres des autres, notamment de populations non européennes ou colonisées. Pensons aux crimes perpétrés contre les Amérindiens, ainsi que les dommages irréversibles faits aux peuples "noirs » à travers la traite négrière, l'esclavage ou la discrimination raciale. S'appesantir lourdement sur la violence subie par une communauté, dans le dessein de définir une identité stricte, scotomise les traumatismes des autres et alimente derechef le sentiment de l'élection, y compris sur le mode de la privation.

\section{La mondialisation des politiques de la mémoire}

La succession de catastrophes et l'ampleur des destructions à la fois humaines et matérielles, culturelles et sociales au $\mathrm{xx}^{\mathrm{e}}$ siècle, ont orienté le devenir des sociétés occidentales (et bien au-delà) vers la condition de victimes. Dès la reconstruction entreprise, à la suite des massacres de masse et des destructions à grande échelle, s'est posée la question de savoir quelle place on devait réserver aux tragédies de vaste amplitude. Que faut-il faire de nos désastres? Faut-il en effacer les traces ou les préserver, les symboliser par le biais d'un dispositif, élever un monument, se limiter à une plaque commémorative, présenter une relique, construire tel ou tel édifice? L'obsession $\mathrm{du}$ souvenir est-elle aussi périlleuse que son refoulement, l'anamnésie aussi problématique que l'amnésie ${ }^{13}$ ? En termes de recouvrement ou de perte, l'alternative est embarrassante: faut-il considérer l'utilité de ces mémoriaux comme la mémoire indélébile du passé ou souhaiter la reconstruction de l'édifice d'origine comme une destruction de la mémoire, comme un rejet probable de l'histoire, comme la prescription d'une amnistie? Aucune législation, aucune déclaration ne permet d'y répondre. Plutôt que de reconstruire ce qui a été détruit, ne faut-il pas mieux laisser la plaie saigner et reposer les corps calcinés? C'est le dilemme qu'avait réglé Chateaubriand (sur un mode romantique) : «Un seul droit, un seul devoir, conserver les ruines à notre admiration, à notre douleur, à notre haine qui viendra s'y alimenter, y renouveler de génération en génération le pieux souvenir." Souhaiter qu'on muséifie les ossements des morts et qu' " on y inscrive le nom de tous les morts en lettres d'or qui feront à l'immense ossuaire le plus beau revêtement qu'aucune imagination puisse rêver» entretient certes l'esthétique kitsch et morbide sans pourtant fournir de réponse décisive (Ash 2003). C'est notamment oublier que la présence des reliques mortuaires devient souvent insupportable à ceux qui y sont quotidiennement confrontés. D'un autre côté, tenir éveillée la mémoire, ne pas l'interrompre est quoi qu'il en soit une nécessité.

Les villes lourdement bombardées pendant la Seconde Guerre mondiale, notamment celle de Dresde, par les Alliés, furent reconstruites. Dubrovnik restaure ses palais, bombardés en 1991 et 1992. L'Unesco a inscrit en 1996, malgré la réprobation des Américains, l'ancien 
pavillon de l'industrie, devenu le Peace Dome d'Hiroshima, sur la liste du patrimoine mondial. Caen a créé son mémorial. Les Khmers préservent intacte l'école qui a servi de centre de détention S-21, dont la mémoire représente effectivement un enjeu crucial pour le Cambodge, comme le rappelle Jean-Louis Margolin dans son article en pointant deux menaces: la spectacularisation de l'horreur et le rejet dans la monstruosité, alors que sont dissimulés les chaînes de responsabilité et le fanatisme idéologique (bien que «l'action des communautés locales et le travail mémoriel de la diaspora puissent participer d'un recours ").

Les architectures qui témoignent le plus intensément des atrocités des années 1940-1944 sont celles qui ont été préservées de la destruction: le corps de garde principal SS, dit "porte de la mort», par lequel les trains de millions de Juifs ont accédé au camp d'Auschwitz II-Birkenau, demeuré intact. Auschwitz-Birkenau fait partie du musée de 200 hectares et de 197 bâtiments que la Diète polonaise approuve en 1947 et que l'Unesco inscrit en 1979 sur la liste du patrimoine mondial. Un musée en plein air. Les pans de mur des maisons, granges, forges et église incendiées et dynamitées du village d'Oradour-surGlane ont été conservés tels quels (quoique réhabilités à date périodique) depuis le 10 juin 1944, puissantes marques du massacre de 642 habitants. Le village est classé par la loi d'exception du 10 mai 1946 monument historique, " pour témoigner au monde entier, à travers le temps, des destructions accumulées sur notre territoire par quatre années d'oppression » et pour autoriser la construction à proximité d'un nouvel Oradour, même si ce voisinage est chaque jour plus difficile à vivre. Un autre musée en plein air.

Soixante ans plus tard, en septembre 2001, Ground Zero, en bas de Manhattan, est devenu un site de destruction et de reconstruction émotionnellement chargé sur un plan local, national et international. Sur le lieu même de la catastrophe, les pratiques de la mémoire et de deuil furent débattues sur un mode à la fois mémoriel, esthétique et politique. L'expérience traumatisante de l'évanouissement des Twin Towers dans les débris matériels et les restes humains exigea qu'on aménage une trace concrète consacrée aux 2 979 victimes. Cette poussière de cendres devint dès lors une substance sacramentelle et cérémonielle qui symbolisait la violence de la perte soudaine. Le site se christianisa progressivement et devint un "espace patriotique sacré", analogue au cimetière de Gettysburg érigé en l'honneur des soldats américains morts pour la nation. Les projets d'y élever un mémorial, flanqué d'un musée abritant des objets (voitures de police, lances d'incendie, bouts d'ossature métallique de la tour nord, etc.), se multiplièrent avec la volonté d'incorporer les ruines pour suggérer le pouvoir destructeur de la haine et rendre simultanément hommage aux victimes transfigurées en "héros combattants»; les exemples du mémorial de la paix d'Hiroshima et de la cathédrale de Coventry furent invoqués. Le site d'urgence devint un lieu de pèlerinage et céda la place au tourisme et aux memorabilia vendus dans des échoppes de souvenirs. Les architectes furent invités à réinventer la structure des tours jumelles en termes postmodernistes, y compris sous la forme de la célébration du vide, mais en maintenant constante la tension entre le respect pour les morts, la mémorisation du traumatisme et la pédagogie de l'extrême (Low 2004 ; Sturken 2004).

Cette quête éperdue d'appropriation et de signification à travers une mise en scène spectaculaire et médiatisée atteste que jamais l'histoire et la mémoire n'ont été aussi présentes dans le devenir des sociétés contemporaines. L'immense champ du souvenir semble d'autant plus en extension que l'éducation par l'émotion historique et mémorielle n'a pas épuisé son capital de mobilisation populaire. Dans tous les États de la planète, les 
exercices et les entreprises de commémoration et de (re)mémoration sont au centre des préoccupations des populations. À vrai dire, il n'est pas un pays qui ne soit maintenant habité par cette question des rapports tumultueux et changeants entre mémoire, histoire et politique. La " politique de la mémoire » marque désormais de manière plus ou moins forte toutes les collectivités contemporaines. Ce qui ne va pas sans risque au regard de l'injonction de ce "devoir de mémoire" qui nourrit le cycle de l'éternelle revanche contre un Autre ennemi, évidemment irréductible. Les membres des communautés risquent surtout d'être vampirisés par les ancêtres au détriment de la liberté des descendants. Le "devoir de mémoire » tel qu'il est pratiqué, c'est-à-dire érigé sous la forme d'un dogme intangible, peut finalement contribuer au renforcement des racismes et de l'antisémitisme tant l'émotion hyperbolique et abstraite dépolitise une histoire fort longue et complexe. Une des raisons rhétoriques qui a favorisé la naissance du musée national des Indiens-Américains (NMAI) dans la ville de Washington (ouvert le 21 septembre 2004) ne le doit qu'au fait qu'il existait déjà là un musée de l'Holocauste consacré aux Juifs. Dans son article sur la représentation du traumatisme dans les musées de la religion et des cultures juives et le NMAI, Reesa Greenberg éclaire les possibilités et les limites des musées qui répondent à une histoire des génocides dans les pays où les victimes et leurs descendants continuent de cohabiter avec ceux qui les ont persécutés et leurs «héritiers", non sans s'interroger sur une série de concepts associés au traumatisme: "se souvenir ", "réparer le monde», "survivance», «résiliance», « orientation temporelle ».

Aux États-Unis, beaucoup soulignent que la destruction des Juifs d'Europe a joué un rôle moindre dans cette partie du monde que l'extermination des Amérindiens; et certains s'étonnent qu'il n'y ait toujours pas de musée de la traite négrière, de l'esclavage ou des discriminations raciales... La vertu de l'enquête conduite par Didier Fassin sur l'expérience du musée de l'Apartheid est de donner vie à une partie de l'histoire de l'Afrique du Sud, en dépit de l'oubli, du déni et de la déréalisation, tout en prenant parti pour l'enseignement des leçons de la ségrégation raciale et de la résistance au régime violemment inégalitaire des Boers (et des Britanniques). Son article montre que la visite du musée s'apparente à une expérience de vérité par laquelle la « rédemption de la nation devient possible ». Dans cette perspective, il serait grand temps que les anthropologues s'inquiètent de l'étourdissant silence qui entoure le fait que les ethnologues aient si peu étudié les dynamiques génocidaires et les pillages des arts dits primitifs alors que leurs « objets » de prédilection furent justement les «survivances (culturelles) et les peuples victimes de répressions sanglantes à la source de nombreux traumatismes, comme l'isolement dans les camps de concentration, sans même parler d'extermination ${ }^{14}$ ».

Rétablir la présence des traumas dans l'agora des États-nations et des communautés doit permettre d'intégrer à l'avenir les enjeux à la fois de l'histoire, de la mémoire et de l'oubli. Cette recréation et cette refondation cherchent à restituer le poids historique de l'événement sous la forme de ces "communautés imaginées » singulières que sont les nations (Anderson 1996). L'enjeu propre au Xxi ${ }^{e}$ siècle est de négocier ou de réinventer l'histoire et la mémoire sur les ruines encore chaudes du passé, au bénéfice d'un avenir à (re)bâtir sur une base revivifiant les acteurs sociaux. Au centre de la négociation ou de la réinvention de ce qui fut soit oublié, soit vécu ou perçu, trône le présent en attente d'inspirer un futur meilleur qui pourrait consolider les assises de la mémoire collective afin de les perpétuer dans l'espace public à travers l'« usage politique de l'histoire », pour employer le vocable de Jürgen Habermas. La mise en scène de la mémoire et de l'histoire 
dans le théâtre de la vie sociale, en particulier dans ses différentes expressions muséales, revêt une dimension cathartique. Loin de référer exclusivement au passé, aux anciens et aux défunts, la mémoire est tout autant, sinon davantage, tournée vers le présent, l'avenir et les descendants. Car l'objectif est bien, comme l'a montré Herbert Hirsch (1995), de transformer les impasses de l'histoire en autant de possibilités pour la suite, du monde et au sein des communautés humaines; de là cette étude de la mort pour préserver la vie.

Cette intention rejoint le questionnement de Paul Ricœur (2000) sur la possibilité de l'humain de réfléchir sur sa condition antérieure dans la perspective de modifier sa condition présente et d'intervenir sur ce que pourraient être une mémoire et une histoire «justes » pour les prédécesseurs et pour les descendants. C'est pour cette raison, selon lui, qu'il faut discuter raisonnablement d'une politique de la «juste mémoire » car le problème de la représentation du passé devient tout à coup écrasant de responsabilité pour les intellectuels aussi bien que pour tout un chacun. Une représentation juste du passé renvoie au respect des faits et des morts mais aussi au point de vue du bonheur des vivants et d'un passage heureux à l'avenir; ce que Paul Ricœur appelle justement la « responsabilité ». Cette question s'avère d'autant plus troublante et angoissante pour l'homme qui cherche à ressaisir son expérience passée et à projeter son horizon d'attente à partir du présent qu'il a un souci général de dépassement des impasses découlant du déroulement et de l'interprétation du passé, « entre le trop de mémoire et le trop d'oubli volontiers incarnés par les excités ou les excédés de la mémoire ». La porte s'avère cependant étroite car nos subjectivités se façonnent désormais dans un monde et au sein de sociétés frappés d'amnésie ou saturés de passé.

41 On observe dans le même temps que la phase contemporaine de la mondialisation a amplifié la fièvre rétrospective en la teintant de «nostalgie" à la fois nationale et impériale. Le musée est devenu dans la foulée une institution sociale majeure. La muséification politique se double d'une mise en musée systématique des vestiges humains et économiques, de la culture matérielle et du terroir historique. L'omniprésence d'un passé que l'on décline tantôt sous forme de commémoration, tantôt dans les termes de la dette, de la repentance et de la réparation remplit et déborde les musées. L'oubli, l'occultation, le déni sont devenus des objets permanents de polémiques. L'allégorie de l'Holocauste, dans les années 1970-1990, les campagnes d'opinion de multiples groupes de pression ont cherché à obtenir la reconnaissance de leur traumatisme en vue d'une éventuelle réparation matérielle ou symbolique.

La reconnaissance par la Turquie du génocide arménien, le négationnisme japonais vis-àvis de la Chine, le sort des personnes «disparues" sous les dictatures militaires en Argentine, en Uruguay et au Chili, ou pendant la dernière guerre civile en Algérie, la rivalité entre communautés se disputant des territoires, des frontières ou des «lieux " marqués par l'histoire comme en Israël ou en Palestine, au Cachemire entre l'Inde et le Pakistan, au Sri Lanka entre Tamouls et Cinghalais, en Bosnie-Herzégovine, mais aussi les revendications « ethniques » et celles liées auxdits "peuples autochtones », la montée en puissance de la question de l'esclavage dans divers pays, y compris en France, et lors de la Conférence mondiale contre le racisme et les discriminations à Durban en 2001, la création des tribunaux pénaux internationaux de La Haye et d'Arusha et de la Cour pénale internationale permanente, la généralisation des commissions "Vérité et réconciliation ", la loi sur la "compétence universelle" de la justice belge sont des exemples parmi d'autres de cette proliférante politique de la mémoire et de son 
institutionnalisation croissante. Le rapport au passé et à la mémoire est devenu un enjeu des relations internationales ainsi qu'un instrument de la "gouvernance globale ». Cette expérience mnémonique généralisée concerne aussi bien les États nationaux postindustriels que les États émergents, pauvres, délités, les organismes transnationaux, les administrations et les ONG, dans un contexte où se multiplient les urgences humanitaires sur une base compassionnelle, le plus souvent dans les anciennes zones coloniales, sans parler des risques de déstabilisation de populations sous le coup d'ingénieries politiques aux effets dévastateurs dans la longue durée.

Finalement, si ce riche dossier d'articles déporte judicieusement au-delà la question muséographique, c'est dans le but de ne pas contribuer à la colonisation du présent tétanisé par le souvenir traumatique sacralisé et sanctuarisé. Ne doutons pas que les chemins de réflexion singuliers empruntés par les auteurs dans le parti pris inébranlable d'«irréconciliation" avec le présent, comme présent des massacres de masse, des violences extrêmes et des camps en diverses régions du monde, apportent un éclairage cristallin à la sismographie des terreurs.

\section{BIBLIOGRAPHIE}

ADORNO,

2006 Métaphysique. Concept et Problèmes. Paris, Payot.

ANDERS, Gunther

2001 L'Obsolescence de l'homme. Sur l'âme à l'époque de la deuxième révolution industrielle. Paris, Encyclopédie des nuisances (1 $1^{\text {re }}$ éd. 1956).

ANDERSON, Benedict

1996 L'Imaginaire national. Réflexion sur l'origine et l'essor du nationalisme. Paris, La Découverte $\left(1^{\text {re }}\right.$ éd. 1983).

Anonyme

2005 Des voix sous la cendre. Manuscrit des Sonderkommado d'Auschwitz-Birkenau. Paris, CalmannLévy.

ARENDT, Hannah

1993 La Condition de l'homme moderne. Paris, Pocket (« Agora ») (1 $1^{\text {re }}$ éd. 1958).

ASH, Timothy Garton

2003 « The Water Of Mesomnesia », in Anne-Marie Le Gloannec et Aleksander Smolar, éds., Entre Kant et Kosovo. études offertes à Pierre Hassner. Paris, Presses de Sciences-Po.

ASSAYAG, Jackie

2004a « La face cachée de la modernité. Anthropologie et génocides », L'Homme 170 : 231-244.

$2004 b$ « Leçons de ténèbres. Violence, terreur, génocides », Les Temps modernes 626 : 275-304. 
2006 «"Prendre le $\mathrm{xx}^{\mathrm{e}}$ siècle à la gorge." Le partage des génocides : spectre, comparaison, colonialisme », L’Homme 177-178 : 469-488.

BENJAMIN, Walter

2000 Euvre III. Paris, Gallimard (« Folio »).

BROSSAT, Alain

1996 L'épreuve du désastre. Le xxe siècle et les camps. Paris, Albin Michel.

CARUTH, Cathy

1996 Unclamed Expérience: Trauma, Narrative, History. Baltimore, The Johns Hopkins University Press.

CHAKRABARTY, Dipesh

2000 Provincializing Europe. Postcolonial Thought and Historical Difference. Princeton - Oxford, Princeton University Press.

2002 « Museums in Late Democracies », Humanities Research IX, 2 (« Museums of the Future », numéro spécial).

CHAUMONT, Jean-Michel

1997 La Concurrence des victimes. Génocides, identités, reconnaissance. Paris, La Découverte.

DELAGE, Christian

2006 La Vérité par l'image. De Nuremberg au procès de Milosevic. Paris, Denoël.

DETIENNE, Marcel

2000 Comparer l'incomparable. Paris, Éditions du Seuil.

DIDI-HUBERMAN, Georges

2004 Images malgré tout. Paris, Éditions de Minuit.

2006 « Ouvrir les camps, fermer les yeux », Annales HSS 5 : 1011-1049.

Finkelstein, Norman G.

2001 L'Industrie de l'Holocauste. Réflexions sur l'exploitation de la souffrance des Juifs. Paris, La F abrique.

FOUCAULT, Michel

1997 Il faut défendre la société. Cours au Collège de France, 1976. Paris, Gallimard.

GELLATELY, Robert, KIERNAN, Ben, éds.

2003 The Specter of Genocide. Mass Murder in Historical Perspective. Cambridge, Cambridge University Press.

GREENBLATT, Stephen

1991 « Resonance and Wonder », in Ivan Karp et Steven D. Levine, éds., Exhibiting Culture.

Washington et Londres, Smithsonian Institution Press : 42-56.

HABERMAS, Jürgen

1985 Frankfurter Allgemeine Zeitung, 22 novembre.

HINTON, Alexander Laban 
2002 Annihilating Difference. The Anthropology of Genocide. Berkeley, The University of California Press.

HIRSCH, Herbert

1995 Genocide and the Politics of Memory: Studying Death to Preserve Life. Chapel Hill (North Carolina), The University of North Carolina Press.

LACAPRA, Dominick

Writing History, Writing Trauma. Baltimore-Londres, The Johns Hopkins University Press.

LEFORT, Claude

1976 Un homme en trop. Paris, Éditions du Seuil.

LEVI, Primo

1993 Lilith et autres nouvelles. Paris, Liana Levi.

Low, Setha M.

2004 « The Memorialization of September 11: Dominant and Local Discourses on the Rebuilding of the World Trade Center site ", American Anthropologist 31, 3 : 326-339.

LYOTARD, Jean-François

1983 Le Différend. Paris, Éditions de Minuit.

MESCHONNIC, Henri

2005 « Israël : pour en finir avec le mot "Shoah" ", Le Monde, 24 février.

MINARD, Pierre

2000 Conscience de la Shoah. Critique des discours et des représentations. Paris, Kimé.

NANCY, Jean-Luc

2001 « La représentation interdite », Le Genre humain 36, «L'art et la mémoire des camps » : 11-38.

NOVICK, Peter

2001 L'Holocauste dans la vie américaine. Paris, Gallimard.

OSIEL, Mark

2006 Juger les crimes de masse. La mémoire collective et le droit. Paris, Éditions du Seuil.

RANCIÈRE, Jacques

2001 "S'il y a de l'irreprésentable », Le Genre humain 36, "L'art et la mémoire des camps » :

81-102.

RICœUR, Paul

2000 La Mémoire, l'histoire, l'oubli. Paris, Éditions du Seuil.

SEMELIN, Jacques

2005 Purifier et détruire. Usages politiques des massacres et génocides. Paris, Éditions du Seuil.

SCHEPER-HUGHES, Nancy, BOURGOIS, Philippe, éds.

2004 Violence in War and Peace: An Anthology. Malden (Massachusetts), Blackwell.

STEINER, George 
1987 « La longue vie de la métaphore », Écrits du temps 14-15 : 4-22.

STURKEN, Marita

2004 « The Æsthetics of Absence: Rebuilding Ground Zero », American Ethnologist 31, $3:$ 311-325.

Weitz, Eric D.

\section{NOTES}

1. Pour un éclaircissement et une discussion critique de la notion des "violences extrêmes ", nous renvoyons à la somme impressionnante sur les génocides publiée par Jacques Semelin (2005).

2. La citation est extraite du livre de Pierre Minard (2000: 149$)$.

3. L'ouvrage de Georges Didi-Huberman (2004) a soulevé une polémique concernant la critique philosophique de l'inimaginable.

4. On emprunte les deux catégories à la fois contradictoires et complémentaires de Stephen Greenblatt définissant les modes poétique et politique de la représentation, qui se croisent lorsque la résonance émerveille et que l'émerveillement résonne.

5. Selon Mark Osiel, le « procès-spectacle démocratique » dans le cadre de la " justice discursive et reconstructive » remplit ces mêmes fonctions en servant de chambre d'écho internationale (Osiel 2006).

6. On a toutes les raisons de rejeter le terme "holocauste ", bien installé aux États-Unis et conforté par la diffusion du film américain du même nom. Non seulement le terme implique une théologie qui justifie le meurtre de masse en le présentant comme une dévotion et un sacrifice en paiement des péchés, ce qui en fait une punition divine, mais c'est aussi parce que c'est un terme grec, qui vient de la traduction de la Septante, texte de base du christianisme. Le consensus s'est déplacé en français sur le mot "Shoah », lui aussi porté par un film à succès (dont l'auteur ignore l'hébreu de son propre aveu). Or le mot n'a rien à voir avec le massacre et n'introduit pas non plus du « providentiel ». Le scandale, c'est d'employer un mot qui désigne un phénomène de la nature pour dire une barbarie toute humaine, ce pourquoi le terme " judéocide » devrait s'imposer. Voir la brillante exégèse philologique et idéologique d'Henri Meschonnic (2005).

7. Voir les ouvrages collectifs dirigés par Alexander Laban Hinton (2002), Robert Gellately et Ben Kiernan (2003) et Nancy Scheper-Hughes et Philippe Bourgois (2004), ainsi que celui d'Eric Weitz (2003) ; sur cette extension et cette tendance, on se permet de renvoyer à la brève synthèse de l'auteur (2006).

8. La notion a été forgée par Emmanuel Levinas dans le cadre de sa réflexion sur le « visage » de l'Autre.

9. L'inventaire de ces principales critiques est résumé dans un article de l'auteur (2004b).

10. On lira avec intérêt son article concernant les musées dans les démocraties modernes (2002).

11. Jean-François Lyotard a formulé le syllogisme sophistique du paradoxe du témoin (qu'utilisent volontiers les « révisionnistes » et les « négationnistes ») : « Pour identifier qu'un local est une chambre à gaz, je n'accepte comme témoin qu'une victime de cette chambre à gaz ; or il ne doit y avoir, selon mon adversaire, de victime que morte, sinon cette chambre à gaz ne serait pas ce qu'il prétend ; il n'y a donc pas de chambre à gaz. » (1983:16-17)

12. «Malgré tout » est le maître mot de l'œuvre subtile et fondamentale de Georges DidiHuberman à propos de l'image ; on se reportera à son article « Ouvrir les camps, fermer les yeux $\gg(2006)$.

13. «Les psychologues ont observé que les survivants d'événements traumatiques se divisent en deux groupes bien définis : ceux qui refoulent leur passé en bloc et ceux dont le souvenir du 
dommage subi persiste, comme gravé dans la pierre, dominant toute l'expérience antérieure et ultérieure ", comme l'a rappelé Primo Levi, qui se classe manifestement dans le second groupe (Levi 1993 : 10-11) ; ce qui vaut pour les hommes vaudrait-il pour les bâtiments?

14. Sur cette indifférence ou négligence des ethnologues et des anthropologues, on se permet de renvoyer à l'article de Jackie Assayag (2004a).

\section{RÉSUMÉS}

$\mathrm{Au}$ refus de porter le regard sur l'événement monstrueux que furent les génocides a succédé la "pédagogie par l'horreur». Dans l'intervalle, il s'est transformé en langage et en objet de consommation «médiatique ». Si le « devoir de mémoire » s'est imposé, la question demeure de savoir ce qu'il faut faire de ces désastres (afin d'éviter que la catastrophe ne se répète). Or il n'y a pas de réponse claire et définitive à cette question. L'essentiel est d'installer une balise ou de tirer un signal d'alarme. Tâche à laquelle s'appliquent les survivants et leurs descendants, ainsi que les résistants ou les militants qui invitent aux commémorations en divers lieux de «mémoires meurtries " : site des crimes, musées, camps, etc. De tels dispositifs montrent en effet que les génocides n'échappent pas à la représentation; que leur singularité ne disqualifie pas la comparaison; que leur définition ne doit pas se profiler exclusivement sur le droit; que le "judéocide" n'est pas une théologie et qu'il ne constitue pas le paradigme des violences extrêmes ; enfin, que les traumatismes doivent être discutés, interprétés et jugés au sein de forums hybrides. En dépit de la montée en puissance des politiques de la mémoire compassionnelle, en voie de mondialisation, il faut donc garantir l'« ininterruption » des récits et des savoirs complexes des désastres et autres crimes de masse.

The refusal to face the horror of genocide has been supplanted by the notion of embracing and using the horror to educate others. In the meantime, it has become a sort of language or media object. While the duty to remember prevails, the question remains of what to do with these horrendous events to ensure they never happen again. There is no clear or set answer to this question, however. The important thing is to sound a warning call, a task taken up by survivors and their descendants, activists and protesters, calling for memorials at various sites of "slaughtered memories": sites of the crimes, museums, camps, etc. Such testaments prove that genocides do not go unrepresented; that their singularity does not preclude comparison; that they must not be defined solely and strictly within the framework of the law ; that "Judaicide" is not a form of theology, nor is it the paradigm of extreme violence ; and, lastly, that the trauma inflicted must be talked about, interpreted and judged in mixed forums. Despite the growing tendency towards compassionate memorials, which is becoming a globalised phenomenon, we must still work to ensure that the complex stories and knowledge arising from genocides and other mass crimes continue uninterrupted.

\section{INDEX}

Mots-clés : génocide, musée, politiques de mémoire, traumatisme, postmodernisme, mondialisation

Keywords : genocide, museum, memory policies, tarumatism, postmodernism, globalization 


\section{AUTEUR}

\section{JACKIE ASSAYAG}

jackie.assayag@ehess.fr. CNRS, Institut interdisciplinaire d'anthropologie du contemporain EHESS 